\title{
A Statistical Model of Head Asymmetry in Infants with Deformational Plagiocephaly
}

\author{
Stéphanie Lanche ${ }^{1,2,3}$, Tron A. Darvann ${ }^{1}$, Hildur Ólafsdóttir ${ }^{2,1}$, \\ Nuno V. Hermann ${ }^{4,1}$, Andrea E. Van Pelt ${ }^{5}$, Daniel Govier ${ }^{5}$, \\ Marissa J. Tenenbaum ${ }^{5}$, Sybill Naidoo ${ }^{5}$, Per Larsen ${ }^{1}$, Sven Kreiborg ${ }^{4,1}$, \\ Rasmus Larsen ${ }^{2}$, and Alex A. Kane ${ }^{5}$ \\ ${ }^{1}$ 3D-Laboratory, (School of Dentistry, University of Copenhagen; Copenhagen \\ University Hospital; Informatics and Mathematical Modelling, \\ Technical University of Denmark), Denmark \\ ${ }^{2}$ Informatics and Mathematical Modelling, Technical \\ University of Denmark, Denmark \\ ${ }^{3}$ Ecole Supérieure de Chimie Physique Electronique de Lyon (ESCPE Lyon), France \\ ${ }^{4}$ Department of Pediatric Dentistry and Clinical Genetics, School of Dentistry, \\ University of Copenhagen, Denmark \\ ${ }^{5}$ Division of Plastic \& Reconstructive Surgery, Washington University School of \\ Medicine, St. Louis, MO, USA
}

\begin{abstract}
Deformational plagiocephaly is a term describing cranial asymmetry and deformation commonly seen in infants. The purpose of this work was to develop a methodology for assessment and modelling of head asymmetry. The clinical population consisted of 38 infants for whom 3-dimensional surface scans of the head had been obtained both before and after their helmet orthotic treatment. Non-rigid registration of a symmetric template to each of the scans provided detailed point correspondence between scans. A new asymmetry measure was defined and was used in order to quantify and localize the asymmetry of each infant's head, and again employed to estimate the improvement of asymmetry after the helmet therapy. A statistical model of head asymmetry was developed (PCA). The main modes of variation were in good agreement with clinical observations, and the model provided an excellent and instructive quantitative description of the asymmetry present in the dataset.
\end{abstract}

\section{Introduction}

Deformational Plagiocephaly (DP) is a term describing cranial asymmetry and deformation commonly seen in infants. Its incidence has been estimated to be as high as $15 \%$ in the USA ([1]). The deformity is thought to result from protracted external intrauterine pressure to the skull, followed by continued postnatal molding due to infant positioning. The incidence has increased exponentially due to the "back to sleep" campaign to promote supine infant positioning to reduce sudden infant death syndrome. DP is manifested most commonly as either leftright asymmetry or brachycephaly (forshortening of the head). Both are treated 
a)

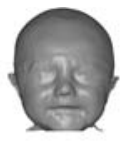

b)

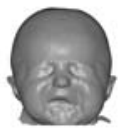

c)

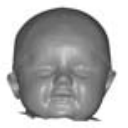

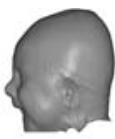
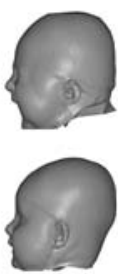
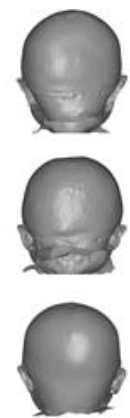
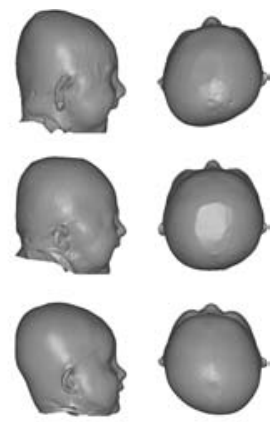

Fig. 1. Five different views of three of the captured 3D full head surfaces. a) Rightsided flattening posteriorly and left-sided flattening anteriorly. b) Brachycephaly. c) Left-sided flattening posteriorly and right-sided flattening anteriorly.

non-surgically. Treatments include parental education on how to prevent further deformation (e.g., alternating sleep positions [2]) and orthotic molding helmet therapy (e.g., 3] and [4). It is widely held that correction is best accomplished in infancy due to the sequence of skull mineralization, however little is known concerning the outcomes from different treatment regimens. DP affects the occiput at the back of the head and, to a lesser extent, the forehead contour. Ear position is often skewed so that the ear is anteriorly positioned on the same side as the occipital flattening. When viewed from above, the head shape can be inscribed within a parallelogram. The purposes of this work were to develop a new methodology for head asymmetry assessment and to develop a statistical model of the asymmetry (using Principal Components Analysis) in order to quantify and localize the asymmetry of each infant's head before and after the helmet therapy and to determine the effect of helmet treatment.

\section{Material}

3D full-head surfaces of 38 patients with DP were captured both before and after treatment utilizing a 3dMD cranial system (www.3dMD.com) at the Division of Plastic \& Reconstructive Surgery, Washington University School of Medicine, St. Louis, MO, USA. All infants commenced their helmet treatments before 6 months of age, and were treated for a maximum of 6 months. Figure 1 presents examples of these scans.

\section{Methods}

\subsection{Template Matching}

The method used for computation and modelling of asymmetry (described in the forthcoming sections) requires establishment of detailed point correspondence between surface points on the left and right sides of the head, respectively. 


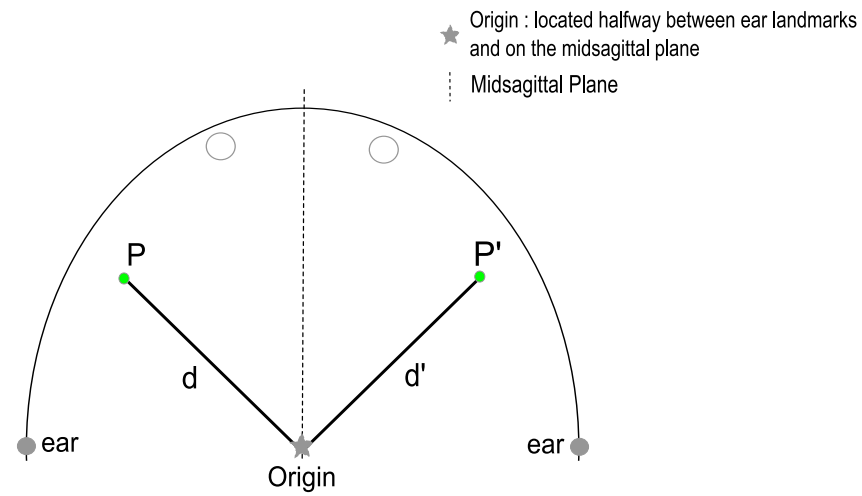

Fig. 2. Computation of the asymmetry: Illustration of the distances $d$ and $d^{\prime}$ between the origin and the points $P$ and $P^{\prime}$, respectively, in an axial view

This is achieved through a process of template matching, whereby a symmetric "ideal" head surface (template) is oriented and deformed to assume the shape of the patient's head surface [5]. The process consists of three steps:

1. Non-isotropic scaling of the template to the patient surface.

2. Rigid orientation of the patient surface to the scaled template surface, using ear-landmarks and nasion.

3. Non-rigid deformation of the scaled template surface to the oriented patient surface using a Thin Plate Spline (TPS) controlled by 22 manually placed facial and ear landmarks, and 40 constructed landmarks on the top of the head. The latter landmarks are determined by intersecting the surfaces with 40 radial lines (equidistant in terms of angle) originating from the midpoint between the ears. They are necessary in order to control the deformation at the top and back of the head where there are no visible anatomical landmarks.

\subsection{Asymmetry Computation}

The definition of the asymmetry $A_{P}$ of a point $P$ involves the computation of the ratio between two distances: 1 ) the distance $d$ from the origin (midpoint between the ear landmarks) to the surface point $P$ on one side of the midsagittal plane, and 2) the distance $d^{\prime}$ from the origin to the corresponding point $P^{\prime}$ on the other side of the midsagittal plane (Figure 2).

Since, intuitively, the amount of asymmetry at $P$ and $P^{\prime}$ should be equal, except for a sign introduced in order to distinguish a point in a "bulged" area from a point in a "flattened" area, $A_{P}$ and $A_{P^{\prime}}$ are defined by:

$$
\begin{aligned}
& \text { if } d>d^{\prime} \text { then } A_{P}=1-\left(\frac{d^{\prime}}{d}\right) \text { and } A_{P^{\prime}}=-A_{P} \\
& \text { if } d^{\prime}>d \text { then } A_{P^{\prime}}=1-\left(\frac{d}{d^{\prime}}\right) \text { and } A_{P}=-A_{P^{\prime}}
\end{aligned}
$$


The change in head asymmetry is calculated as the difference between the asymmetry absolute values at the two stages:

$$
\text { Change }=\left|A_{P, \text { stage } 1}\right|-\left|A_{P, \text { stage } 2}\right|
$$

Hence, a positive change (improvement) implies that $A_{P, \text { stage } 2}$ is closer to 0 than

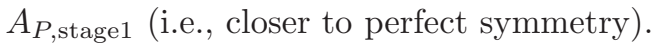

\subsection{Modelling Asymmetry Using Principal Components Analysis}

PCA is a popular method for shape modelling (an excellent description is found in [6]). The PCA is performed as an eigenanalysis of the covariance matrix of the (aligned) asymmetry measures.

The asymmetry values for each scan are ordered according to the mesh points of the template scan (cf section 3.1.) and stored in a vector of size $M$ :

$$
\mathbf{a}=\left[\left|A_{P 1}\right|,\left|A_{P 2}\right|, \ldots,\left|A_{P M / 2}\right|,\left|A_{P^{\prime} 1}\right|,\left|A_{P^{\prime} 2}\right|, \ldots,\left|A_{P^{\prime} M / 2}\right|\right]
$$

Here the first and last $M / 2$ elements are asymmetry values for the points on the right and left sides of the midsagittal plane, respectively. The maximumlikelihood estimate of the covariance matrix can be written as:

$$
\boldsymbol{\Sigma}_{a}=\frac{1}{N} \sum_{i=1}^{N}\left(\mathbf{a}_{i}-\overline{\mathbf{a}}_{i}\right)\left(\mathbf{a}_{i}-\overline{\mathbf{a}}_{i}\right)^{\mathrm{T}}=\frac{1}{N} \mathbf{A} \mathbf{A}^{\mathrm{T}}
$$

where $\overline{\mathbf{a}}$ is the maximum-likelihood estimate of the mean asymmetry of the $\mathrm{N}$ data-vectors. The principal axes of the M-dimensional point cloud of asymmetry are now given as eigenvectors, $\boldsymbol{\Phi}_{a}$, of the covariance matrix:

$$
\boldsymbol{\Sigma}_{a} \boldsymbol{\Phi}_{a}=\boldsymbol{\Phi}_{a} \boldsymbol{\Lambda}_{a}
$$

where $\boldsymbol{\Lambda}_{a}$ is a diagonal matrix containing the eigenvalues of the covariance matrix, and the columns of $\boldsymbol{\Phi}_{a}$ contain its eigenvectors. An asymmetry instance can be generated by modifying the mean asymmetry by adding a linear combination of eigenvectors:

$$
\mathbf{a}=\overline{\mathbf{a}}+\boldsymbol{\Phi}_{a} \mathbf{b}_{a}
$$

where $\mathbf{b}_{a}$ is a matrix containing the asymmetry model parameters.

As the number of observations $(N=76$ scans $)$ is much smaller than the number of surface points $(M=190076)$, the eigenanalysis is carried out using a reduced covariance matrix:

$$
\boldsymbol{\Sigma}_{r e d u c}=\frac{1}{N} \mathbf{A}^{\mathrm{T}} \mathbf{A}
$$

The eigenanalysis of this matrix gives the eigenvalues and eigenvectors of the covariance matrix. The eigenvalues and the eigenvectors may then be computed by:

$$
\begin{aligned}
\boldsymbol{\Lambda}_{a} & =\boldsymbol{\Lambda}_{\text {reduc }} \\
\boldsymbol{\Phi}_{a} & =\mathbf{A} \boldsymbol{\Phi}_{\text {reduc }}
\end{aligned}
$$

In practice, the eigenanalysis may be carried out by Singular Value Decomposition (SVD). 


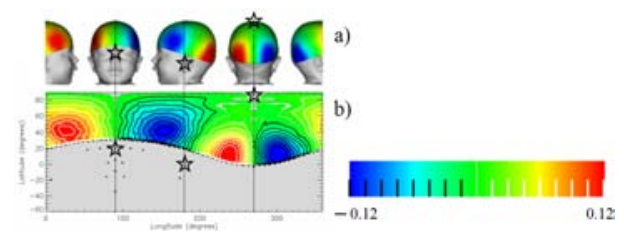

Fig. 3. Flat map construction. a) Asymmetry values in an example subject shown as color coding. b) Corresponding flat map with contours (black: negative, white: positive). Some landmarks are shown as star symbols. Lower limit of helmet region is shown as dashed curve.

\subsection{Projection of 3D Surfaces into 2D Flat Maps}

A more compact means of presentation is to construct a flat map (Figure 3 b) by a simple transformation from rectangular to spherical coordinates. The flat map has right ear landmark at longitude $=0$ degrees, midface at 90 degrees, left ear landmark at 180 degrees and center of the back of the head at 270 degrees. Regions below the helmet area are shown in light gray, below the dashed curve. Levels of asymmetry are indicated by contours in the flatmap. There are 16 contour intervals, spanning the range of asymmetry as indicated by the color bar. The contours are equidistant in terms of asymmetry and are drawn in black for negative values, and in white for positive values. Hence, black contours show "bulged" areas (negative), white contours "flattened" areas (positive), and areas exhibiting no asymmetry are displayed in light gray.

\section{Results}

\subsection{Asymmetry}

Figure 4 presents the results of the asymmetry computations in three example subjects. Top views of the head before (a) and after (b) treatment are shown together with corresponding asymmetry flat maps. In addition, a map of change (c) is shown.

Figure 41. shows an asymmetric DP patient with right-sided flattening posteriorly, as well as a left-sided flattening anteriorly (a). The typical parallelogram shape is also reflected in the asymmetry flat map. Note the improvement in asymmetry after treatment (b,c).

Figure 4.2. shows a typical brachycephalic patient (a). Brachycephalic patients are generally not very asymmetric, as their deformation mainly causes a foreshortning of the skull. Note improved shape after treatment (b,c).

The third patient, Figure 4.3, has left-sided flattening posteriorly as well as a right-sided flattening anteriorly (a). Note the improvement after treatment $(\mathrm{b}, \mathrm{c})$. 


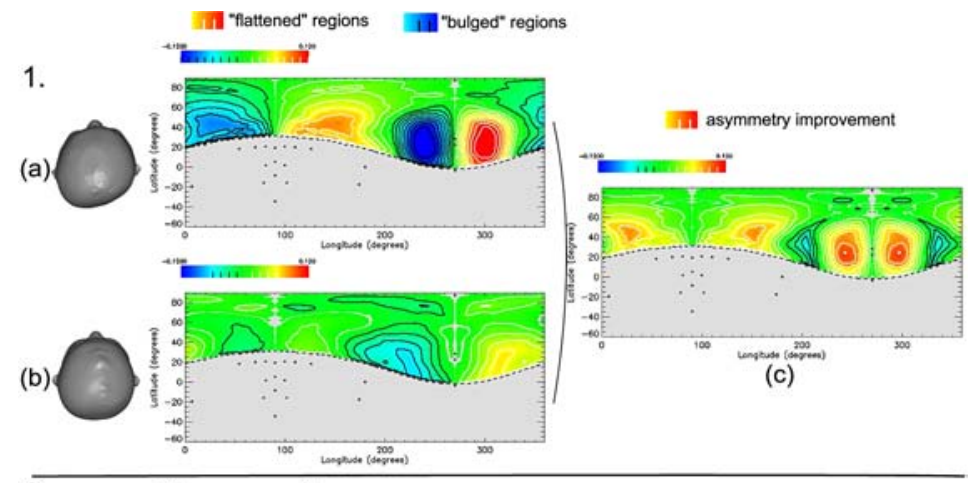

\section{2.}

(a)
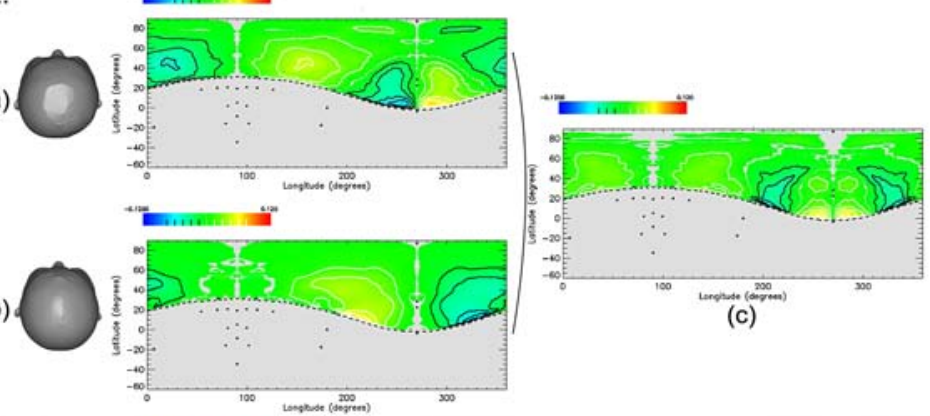

(b)

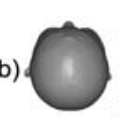

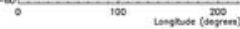

(c)

\section{3.}

(a)
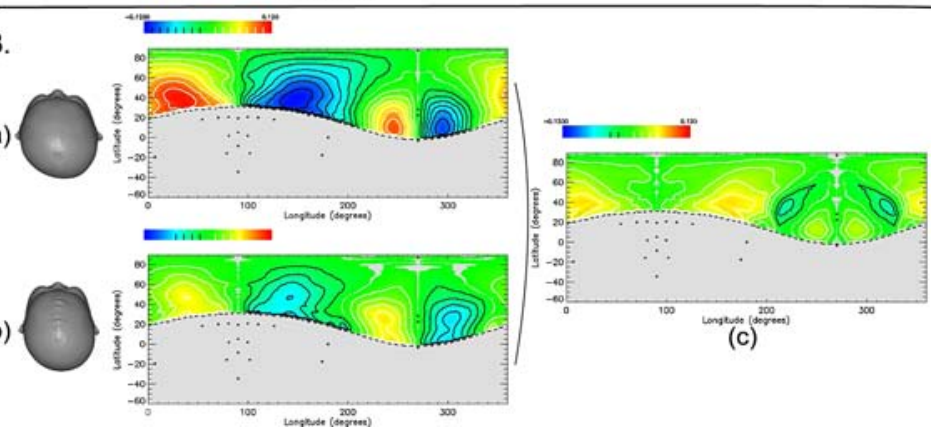

(c)

Fig. 4. Results of the asymmetry computation and changes for: 1. Right-sided flattening posteriorly and left-sided flattening anteriorly. 2. Brachycephaly. 3. Left-sided flattening posteriorly and right-sided flattening anteriorly. (a) Scans at stage 1. (b) Scans at stage 2. (c) Changes between the two stages. In the flat maps showing asymmetry (middle column), positive and negative values denote "flattening" and "bulging" respectively. In the flat maps of change, positive values denote improvement.

\subsection{Statistical Model}

A statistical model was created by performing PCA on the 76 scans. The input of the PCA was the vector of asymmetry measures at each point in the helmet region. The decay of eigenvalues (Figure 5 a) indicates that $96 \%$ of the asymmetry variation can be modelled using the first eight parameters. The mean 
asymmetry (Figure 5b) emphasizes posterior and anterior regions with high asymmetry, while the anterior parts exhibit smaller magnitude.

Figures 5 (c-j) display the first eight modes showing only $\mathbf{\Phi}_{a}$ (cf. Equation 17) with $\mathbf{b}_{a}=-3$ standard deviations. As the images corresponding to $\mathbf{b}_{a}=+3$ standard deviations are exactly the same as $\mathbf{b}_{a}=-3$ standard deviations but with opposite colors, they are not displayed. The first mode (c) localized the main asymmetry variation to the posterior region of the head. The second mode (d) represents variations occuring in the anterior region of the head, but spatially more spread out than the posterior region. The variations of the third mode occured above the ears, also seen in Figure 4. Modes four (f) and five (g) revealed variability mainly in the posterior area of the head, probably the result of variation in the location of the affected area posteriorly. In general, higher modes represented higher spatial frequencies of variation.

The scores of the three first modes (Figure 6) demonstrate the direction and amount of asymmetry progress for each individual. In Figure 6a, the scores for PC2 are plotted against the scores for PC1. The amount of posterior and anterior asymmetry may be read off the $x$ - and $y$-axes, respectively. The least amount of asymmetry is found in the upper-left corner of this figure. This is the region where good treatment outcomes are located, as well as the brachycephalic heads. Individuals that improve in terms of posterior asymmetry move leftwards in the diagram, whereras individuals that improve in terms of anterior asymmertry move upward. Analogously, in Figure 6 $\mathrm{b}$, individuals that improve in terms of asymmetry above the ear move downward.

\subsection{Validation of the Asymmetry Model}

The usefulness of the asymmetry model depends on its ability to capture and describe clinically relevant information in a compact way. Two of the most important parameters describing head asymmetry in DP could be stated as "magnitude of posterior asymmetry" and "magnitude of anterior asymmetry". In the previous section there was strong evidence that the first two modes were related to these particular clinical parameters. To check the strength of the relation between the model modes and the clinical parameters, a search for local extrema of asymmetry was conducted in the asymmetry flat maps. Figure 7 shows the correlation between scores and local minima.

\section{Discussion}

The computed asymmetry corresponded well (Figure 4) to observed asymmetry in the scans. Limitations of the method of establishing point correspondence between scans were the use of the ears (that are often affected in DP) for the registration, and the use of constructed landmarks instead of anatomical landmarks on top of the head. None of these limitations seem to have severely affected a valid asymmetry measurement. PCA is often used for summarizing data. The new variables created by PCA, however, are not guaranteed to be interpretable. 
(a)

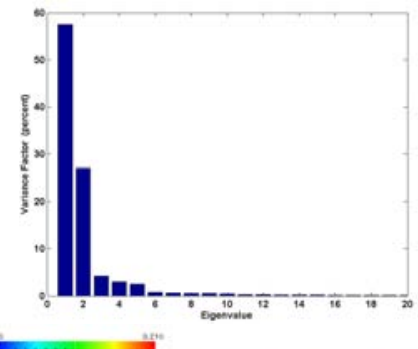

(c)

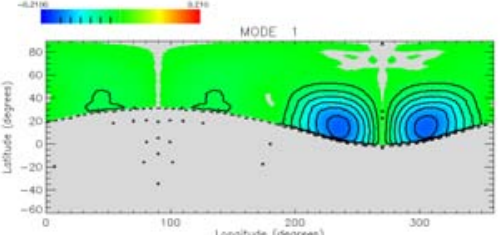

(e)

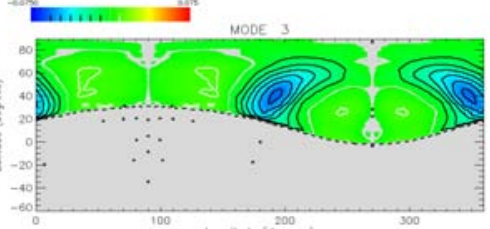

(g)
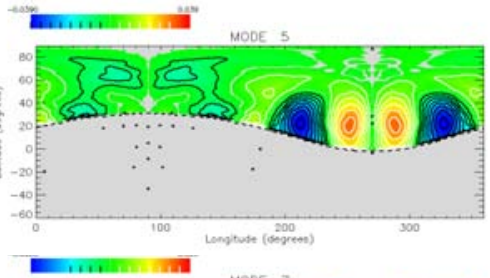

(i)

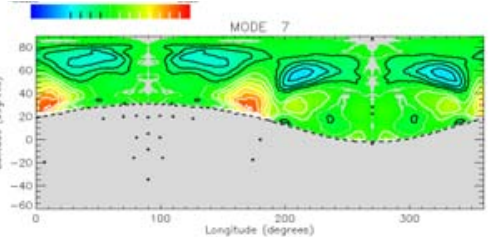

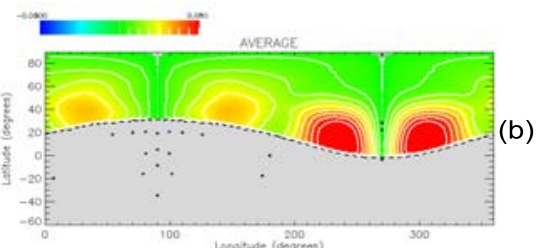
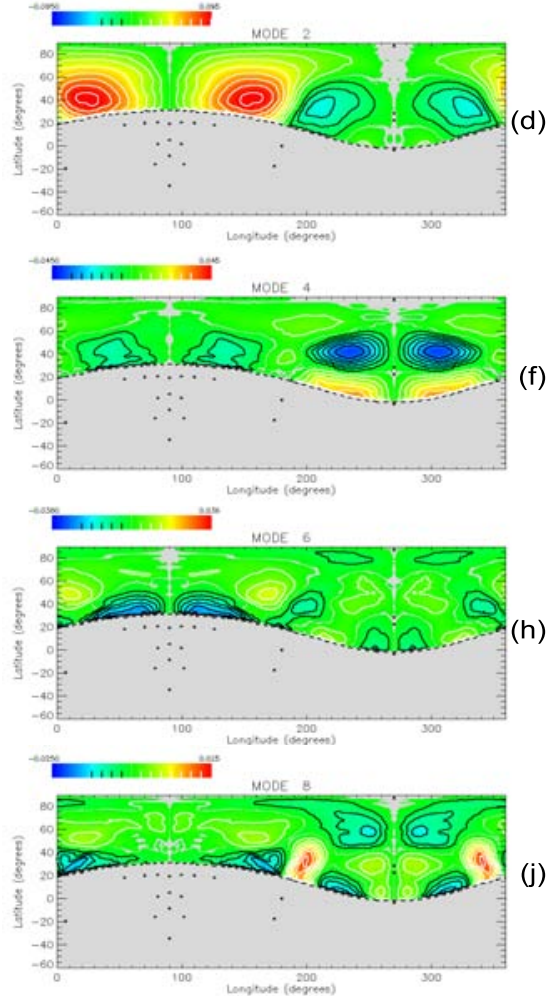

Fig. 5. Presentation of the asymmetry model. (a) Eigenvalues (as percentage of the total variation). (b) Mean asymmetry. (c)-(j) Modes 1 to 8. Modes are shown as variation at -3 standard deviations from the mean. Within the same mode, regions displayed with opposite contour colors (black and white) vary in opposite directions.

The success of the asymmetry model (Figure 7) could be due to the less complex, "global" types of asymmetry variation present in the DP dataset. The excellent properties of the model makes using the model attractive compared to other methods of asymmetry assessment. Other methods, as direct anthropometry of the head (e.g., 7]), measurement systems using a head ring or strip (e.g., 8], and [9]), or even measurements on 3D scans (e.g., 10]), produce a multitude of parameters, making the interpretration difficult in terms of asymmetry and 

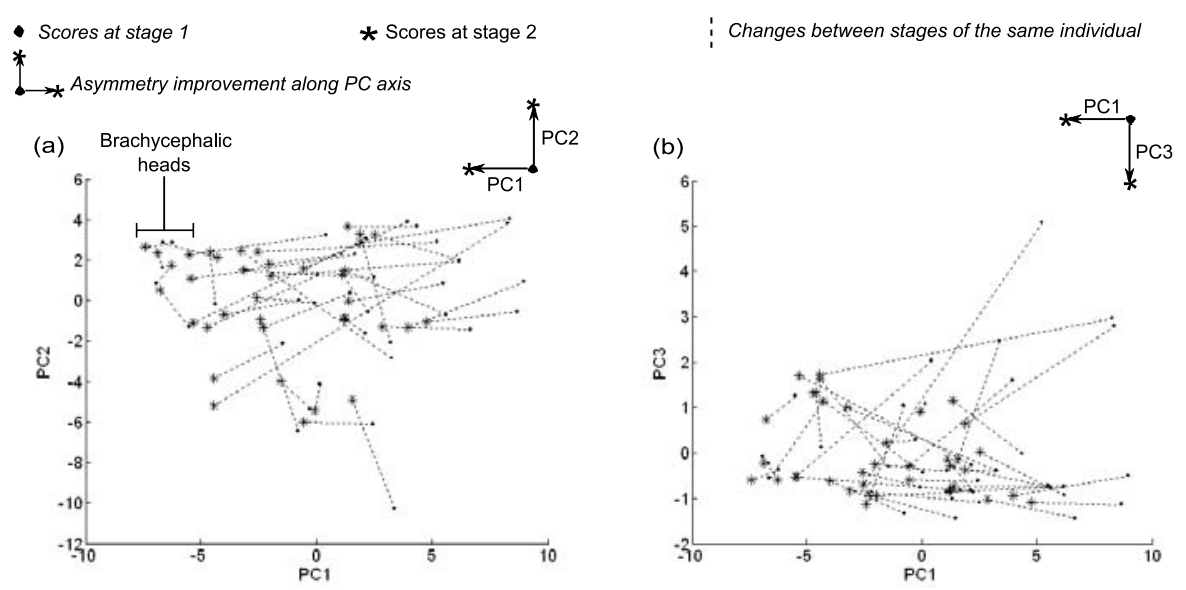

Fig. 6. Score plots of the asymmetry model: (a) PC1 vs. PC2. (b) PC1 vs. PC3.
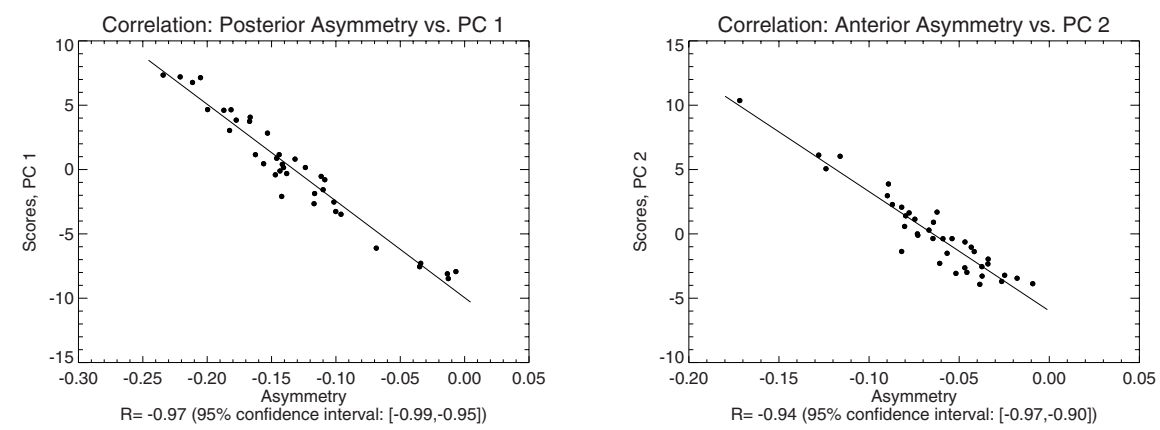

Fig. 7. Correlation between clinical parameters and model PC scores

less intuitive. Contrary to 11 and 12, which use a sparse set of inter-landmark distances, computing the asymmetry at every surface point provides the opportunity to create a high spatial resolution asymmetry model.

\section{Conclusion}

A new 3D asymmetry measure was developed, providing a detailed surface map of asymmetry covering the whole head. The asymmetry measure was seen to reflect observed asymmetry in DP very well. A statistical model was created by performing PCA on the asymmetry maps in 38 patients. PCA modes were seen to correspond very well to clinically relevant parameters. In particular, the first and second modes corresponded to variation at the back and front of the head, respectively. The method is suitable for monitoring asymmetry treatment in individuals, as well as for classifying asymmetry in population studies.

SL acknowledges financial support from the BIOP graduate school. 


\section{References}

1. Littlefield, T.R.: Cranial remodeling devices: treatment of deformational plagiocephaly and postsurgical applications. Semin. Pediatr. Neurol. 11, 268-277 (2004)

2. Hummel, P., Fortado, D.: A parents' guide to improving head shape. Adv. Neonatal Care 5, 341-342 (2005)

3. American Academy of Pediatrics Task Force on Sudden Infant Death Syndrome. Pediatrics vol. 116, pp. 1245-1255 (2005)

4. Lee, W.T., Richards, K., Redhed, J., Papay, F.A.: A pneumatic orthotic cranial molding helmet for correcting positional plagiocephaly. J. Craniofac. Surg. 17, 139$144(2006)$

5. Darvann, T.A., Hermann, N.V., Tenenbaum, M.J., Govier, D., Naidoo, S., Larsen, P., Kreiborg, S., Kane, A.A.: Head shape development in positional plagiocephaly: Methods for registration of surface scans. In: proceedings: Darvann, T.A., Hermann, N.V., Larsen, P., Kreiborg, S. (eds.): "Craniofacial Image Analysis for Biology, Clinical Genetics, Diagnostics and Treatment", Workshop of the 9th MICCAI conference, Copenhagen, Denmark, pp. 59-66 (October 5) (2006)

6. Stegmann, M.B.: Active Appearance Models. IMM Technical Report, IMM-EKS 2000-25, Technical University of Denmark, Lyngby, Denmark (2000)

7. Kolar, J.C., Salter, E.M.: Craniofacial Anthropometry. Practical Measurement of the Head and Face for Clinical, Surgical and Research Use. Springfield, Illinois: Charles C. Thomas, Publisher (1997)

8. Chang, P.Y., Chang, N.C., Perng, D.B., Chien, Y.W., Huang, F.Y.: Computeraided measurement of cranial asymmetry in children with and without torticollis. Clin. Orthod. Res. 4, 200-205 (2001)

9. van Vlimmeren, L.A., Takken, T., van Adrichen, L.N.A., van der Graaf, Y., Helders, P.J.M., Engelbert, R.H.H.: Plagiocephalometry: a non-invasive method to quantify asymmetry of the skull; a reliability study. Eur. J. Pediatr. 165, 149-157 (2006)

10. Plank, L.H., Giavedoni, B., Lombardo, J.R., Geil, M.D., Reisner, A.: Comparison of infant head shape changes in deformational plagiocephaly following treatment with a cranial remolding orthosis using a noninvasive laser shape digitizer. J. Craniofac. Surg. 17(6), 1084-1091 (2006)

11. Lele, R., Richtsmeier, T.: An Invariant Approach to Statistical Analysis of Shapes. Chapman \& Hall/CRC (2001)

12. Bookstein, F.: Morphometric Tools for Landmark Data. Cambridge (1997) 